\title{
Design of Spread Spectrum TT\&C Link for UAV-Swarm Based on Multi - path
}

\author{
Pan Jinglong ${ }^{1, a^{*}}$, Zheng Linhua ${ }^{1}$, Li Hua ${ }^{1}$, Wang $\operatorname{Tan}^{1}$ \\ ${ }^{1}$ School of Electronic Science and Engineering, NUDT, Changsha, Hunan, China \\ a panjingl@foxmail.com \\ *corresponding author
}

Keywords: UAV-Swarm, DS Spread Spectrum, TT\&C, Rake receiver

\begin{abstract}
In this paper, a kind of TT\&C link is designed for the TT\&C system of UAV-Swarm, and the characteristics of code division multiple access are used to realize the TT\&C of the mass unmanned aerial vehicle. Due to the effects of noise and multipath fading in mobile wireless channels, multipath fading can enhance the received signal power through Rake receiver technology and improve the reliability of system transmission by equal gain combining.Under the condition of low SNR, the relatively low bit error rate can be achieved.
\end{abstract}

\section{Introduction}

The UAV-Swarm refers to the unmanned aerial vehicle equipped with radar, photoelectric detection equipment and fire strikes weapons, each machine through the data link between the data exchange and integration, can be deployed more than one unmanned aerial vehicle cluster [1]. The UAV single function is simple, the control is easier, and the machine is small and scattered, the enemy is difficult to all detect and locate, destroy, and pay the attack price will be high [2].

One-station multi-machine data link technology is one of the key technologies in UAV TT\&C system. Multiple access mode is an effective means to achieve multi-machine monitoring and control [3-5].The reference [4] introduced a TDMA-based multi-UAV TT\&C system, but in highspeed digital communication systems, the symbol is too narrow need more advanced equalization measures, and accurate timing and synchronization also requires complex technology. The control station through the code division multiple access way to distinguish between different UAV telemetry parameters and sensor information, the British company has developed the use of spread spectrum technology multi-UAV control system (MUMACS), can also 4 UAV for telemetry, remote control [5].

And different from land mobile communication, UAV TT\&C stations are using directional highgain TT\&C antenna. Therefore, there is a strong direct vision signal LOS (Line of sight) component and a certain Gaussian white noise in the UAV communication system. In the communication link, there are also multipath components caused by different propagation paths in the UAV TT\&C system, similar to the Rice channel [6]. Rake receiver technology can be in time to distinguish the fine multipath signal, so that complex into a strengthened signal. In this paper, the Rake receiver technology is used to realize the TT\&C of the colony unmanned aerial vehicle under the condition of considering the multipath effect. In the end of the paper the design and control link in the low signal to noise ratio can achieve a lower bit error rate.

\section{Control link system design}

\subsection{Transmitter design}

In the TT\&C system, the signal is transmitted in the form of a frame in the channel, the frame structure varies with the channel type and the data rate.

As shown in Figure 1, it is the frame structure of the control traffic channel. Where F denotes the frame header and $\mathrm{T}$ denotes the encoder trailing bit. Transmission rate of $9600 \mathrm{bps}$, the $20 \mathrm{~ms}$ frame 
duration can send 192bit. This 192bit consists of 164 information bits, 12 frame header bits, 8 frame guard bits, and 8 coded trailing bits.

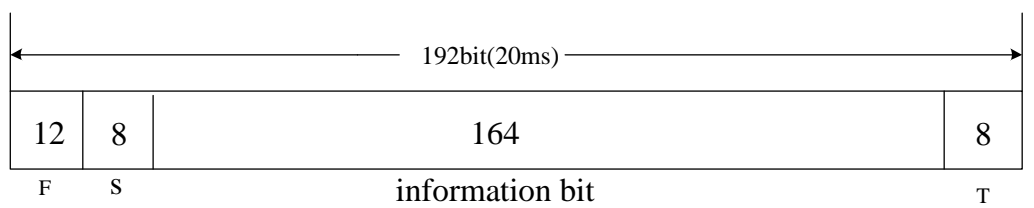

Figure 1 Frame structure of 9600 bps for TT\&C traffic channel

According to the block diagram of the control traffic channel, the system design block diagram of the transmitter part is shown in Figure 2.

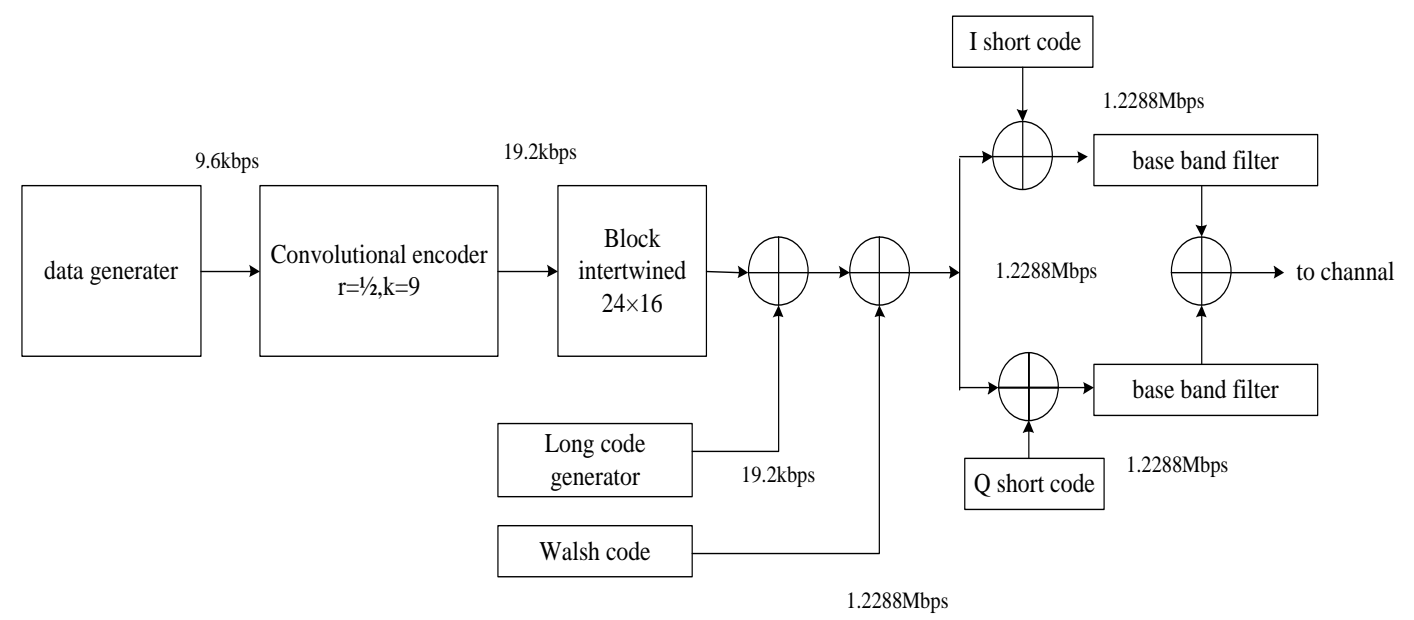

Figure 2 Block diagram of the transmitter system

The data generator generates data at 192 bits per frame as the source of the system. After the convolutional encoder with a bit rate of $1 / 2$ and a constraint length of 9 , the number of modulation symbols per frame is $192 \times 2=384$. The block interleaver treats the input data as a 16-column by 24-row matrix and processes 384 symbols in one frame at a time. In order to provide confidentiality, the interleaved data is compared with the long code generator generated by the long code generator to scramble the data. One of the two orthogonal 64 Walsh sequences is modulo 2 in the data stream to provide uniform RF carrier multiplexing. Two-way short code as I/Q orthogonal sequence of data spread spectrum, modulation using QPSK, after the baseband filter, I, Q two signals to be sent to the signal sent to the channel.

\subsection{1. convolutional coding}

The convolutional code is generated by sending a sequence of transmitted information through a linear, finite state shift register. Typically, the shift register consists of $\mathrm{k}$-level ( $\mathrm{k}$ bits per level) and $\mathrm{n}$ linear algebraic functions. The binary data shift is input to the encoder, moving the $\mathrm{k}$ bits along the shift register each time. Each k-bit long input sequence corresponds to an n-bit long output sequence. So its coding path is defined as $\mathrm{Rc}=\mathrm{k} / \mathrm{n}$. The parameter $\mathrm{k}$ is called the constraint length of the convolution.

It can be seen from the monitoring link channel diagram that the convolutional code rate is $1 / 2$ and the constraint length is $\mathrm{k}=9$. The schematic of the encoder is shown in Figure 3. The generation function of this code is

$$
\begin{aligned}
& \text { g0 }=(1111101011)=(753) \mathrm{o} \\
& \text { g1 }=(1011100001)=(561) \mathrm{o}
\end{aligned}
$$

For each data bit input to the encoder, two code symbols are generated. The code symbols should be output such that the code symbol $\mathrm{c}_{0}$ encoded by the generation function $\mathrm{g}_{0}$ is outputted first, and 
the code symbol $\mathrm{c}_{1}$ encoded by the generation function $\mathrm{g}_{1}$ is output. At the time of initialization, the convolutional encoder should be all zeros. The first code symbol after initialization should be encoded by the generator function $\mathrm{g}_{0}$.

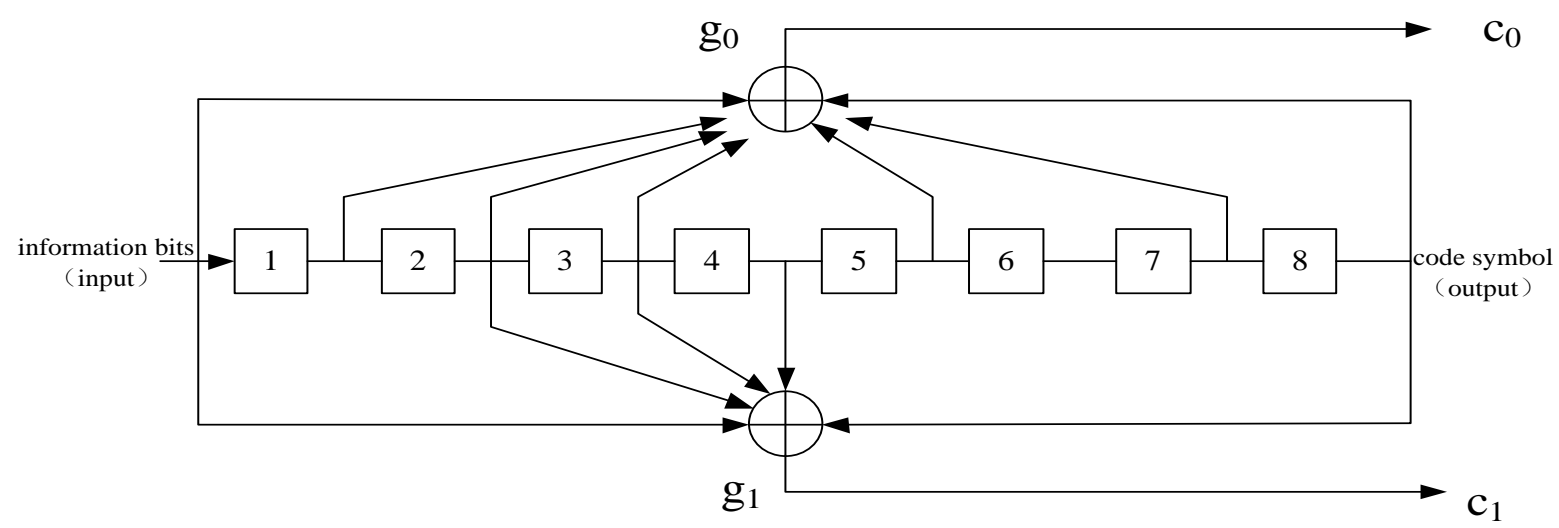

Figure 3 Convolutional encoder: $r=1 / 2, k=9$

\subsection{2. block interleaved}

Interweaving is often combined with coding and is a time-diversity form of square box burst errors. The symbols are changed in order or interleaved before entering the burst channel. If the transmission is a sudden error, restore the original sequence can be scattered in time error. If the interleaver is well designed, the errors will be randomly distributed, and coding techniques will be easier to correct.

A (I, J) block interleaver can be viewed as a storage matrix with a row J column. Data is written in columns and read by row. The symbol starts writing from the upper left corner of the matrix and starts reading from the lower right corner. Continuous data processing requires two matrices: one for data write and the other for data readout. The deinterleaving process also requires two matrices to reverse the interleaving process. 9.6kbps TT\&C channel in a 20ms, 384 symbols on the frame shown in Figure $4(16,24)$ output matrix, the input matrix in order to write the interleaver.

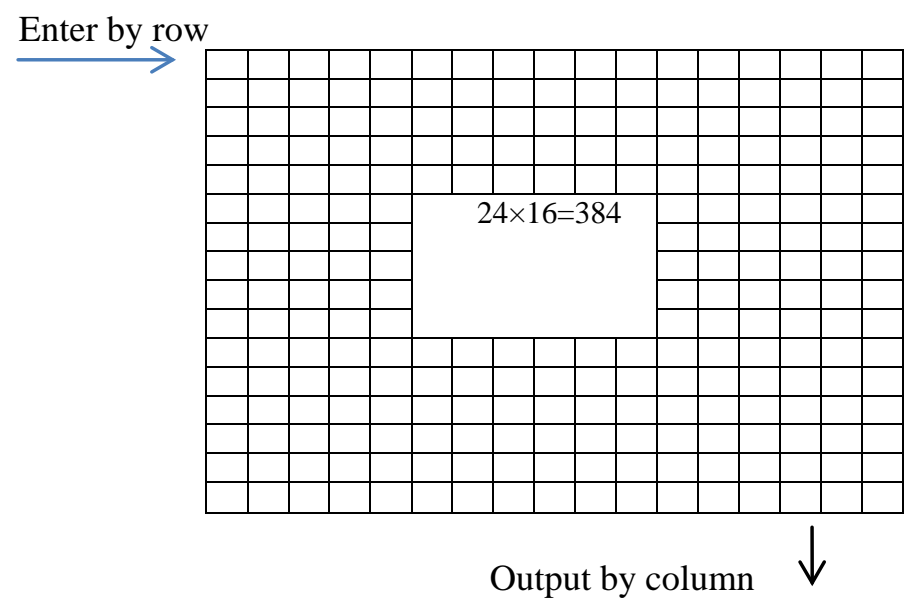

Figure 4 Interleaver output sequence for 9.6 kbps monitoring and control channels

\subsubsection{Data Scrambling}

One of the main problems of wireless communication is that any transmission can be easily disturbed by the enemy. In order to enhance the confidentiality of transmission, the scrambling process adds the genetic code to the outgoing data. The encoding process is done with a key called a long code. Only to know the correct value for the initial value of the base, the receiver can rush to build a long code and decrypt the message.

The rate of the long PN code sequence is $1.2288 \mathrm{Mbps}$, and the rate is reduced to $19.2 \mathrm{kbps}$ by sampling the $64 \mathrm{PV}$ chips at a time. The long PN code is generated with a 42-order shift register 
with a period of $242-1 \approx 3.4 \times 1012$ chips (which lasts for 41 days at a rate of $1.2288 \mathrm{Mcps}$ ). The linear recursion is based on the characteristic polynomial:

$$
\begin{aligned}
& \mathrm{p}(\mathrm{x})=\mathrm{x}^{42}+\mathrm{x}^{35}+\mathrm{x}^{33}+\mathrm{x}^{31}+\mathrm{x}^{27}+\mathrm{x}^{25}+\mathrm{x}^{22}+\mathrm{x}^{21}+\mathrm{x}^{19}+\mathrm{x}^{18}+\mathrm{x}^{17}+\mathrm{x}^{16}+\mathrm{x}^{10} \\
& +\mathrm{x}^{7}+\mathrm{x}^{6}+\mathrm{x}^{5}+\mathrm{x}^{3}+\mathrm{x}^{2}+\mathrm{x}+1
\end{aligned}
$$

As shown in Figures 5, each chip of the long code is generated by a 42-bit mask with a 42-bit state vector of the sequencer.

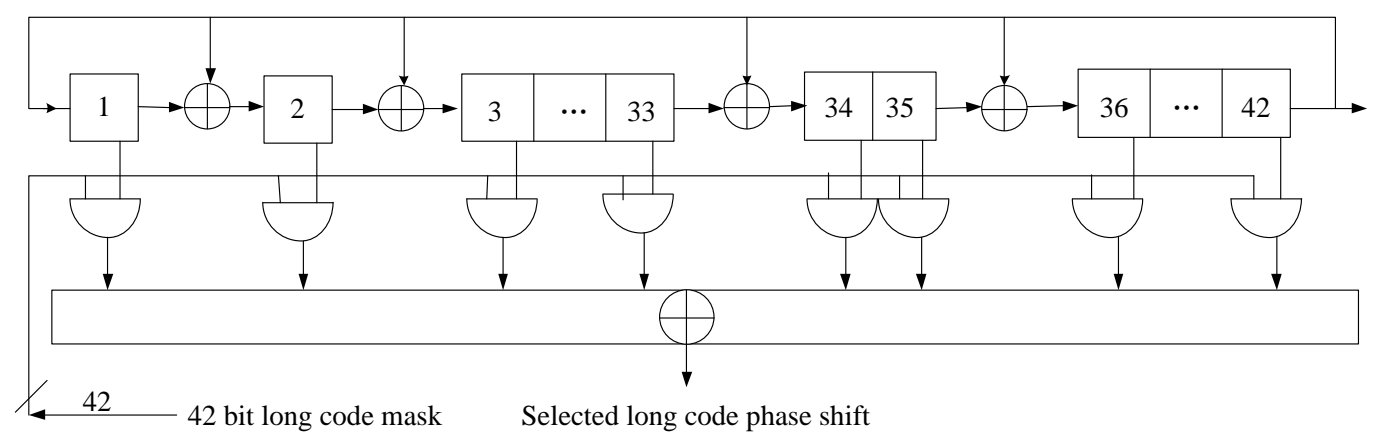

Figure 5 Long code generator

\subsubsection{Orthogonal multiplexing}

In the TT\&C link, each channel through its dedicated orthogonal Walsh sequence is different from other channels. The channel of the TT\&C link consists of a pilot channel, a synchronization channel, a paging channel and a traffic channel. Each channel is modulated by a channel-specific Walsh sequence, and the Walsh sequence is $\mathrm{H}_{\mathrm{i}}$, where $\mathrm{i}=0,1, \ldots, 63$. We assign $\mathrm{H} 0$ to the pilot channel, assign $\mathrm{H}_{32}$ to the sync channel, $\mathrm{H}_{1}$ to $\mathrm{H}_{7}$ to the paging channel, and the rest of $\mathrm{H}_{\mathrm{i}}$ assigned to the traffic channel.

The Walsh sequence has a dimension of 2 for a row in the Hadamard matrix, and when they are correlated over a period of length, they are orthogonal. The 2N-order Hadamard matrix can be generated by the recursive formula:

$$
H_{1}=1, \quad H_{2}=\left[\begin{array}{cc}
1 & 1 \\
1 & -1
\end{array}\right], H_{2 \mathrm{~N}}=\left[\begin{array}{ll}
H_{N} & H_{N} \\
H_{N} & H_{N}
\end{array}\right]
$$

We define $\overline{H_{N}}$ is $H_{N}$ takes negative (for its complement).

In the traffic channel, each input bit in the $19.2 \mathrm{kbps}$ data stream is modulo-2 plus the 64-order Walsh sequence, mapped to 64 output bits. Thus, the output rate of this process is $1.2288 \mathrm{Mbps}$.

\subsubsection{Orthogonal Spread Spectrum}

We use two modified short PN sequences to spread the QPSK in-phase and quadrature branches. The two short PN codes are the m sequences generated by the 15th order shift register, and each cycle inserts an extra ' 0 ' at a specific position of the PN sequence. So the corrected short PN code period is $215=32786$ chips. The sequence is called a guided PN sequence, which is used to identify different TT\&C stations. Different measurement stations use the same pilot PN sequence, but each uses a different phase offset.

The characteristic polynomial of the short PN code used by the inphase branch (I) is:

$$
\mathrm{P}_{\mathrm{I}}(\mathrm{x})=\mathrm{x}^{15}+\mathrm{x}^{13}+\mathrm{x}^{9}+\mathrm{x}^{8}+\mathrm{x}^{7}+\mathrm{x}^{5}+1
$$

The characteristic polynomial of the short PN code used in the orthogonal branch $(\mathrm{Q})$ is:

$$
\mathrm{P}_{\mathrm{Q}}(\mathrm{x})=\mathrm{x}^{15}+\mathrm{x}^{12}+\mathrm{x}^{11}+\mathrm{x}^{10}+\mathrm{x}^{6}+\mathrm{x}^{5}+\mathrm{x}^{4}+\mathrm{x}^{3}+1
$$

As shown in Figure 6, a template is used to generate a short phase of a particular phase. 

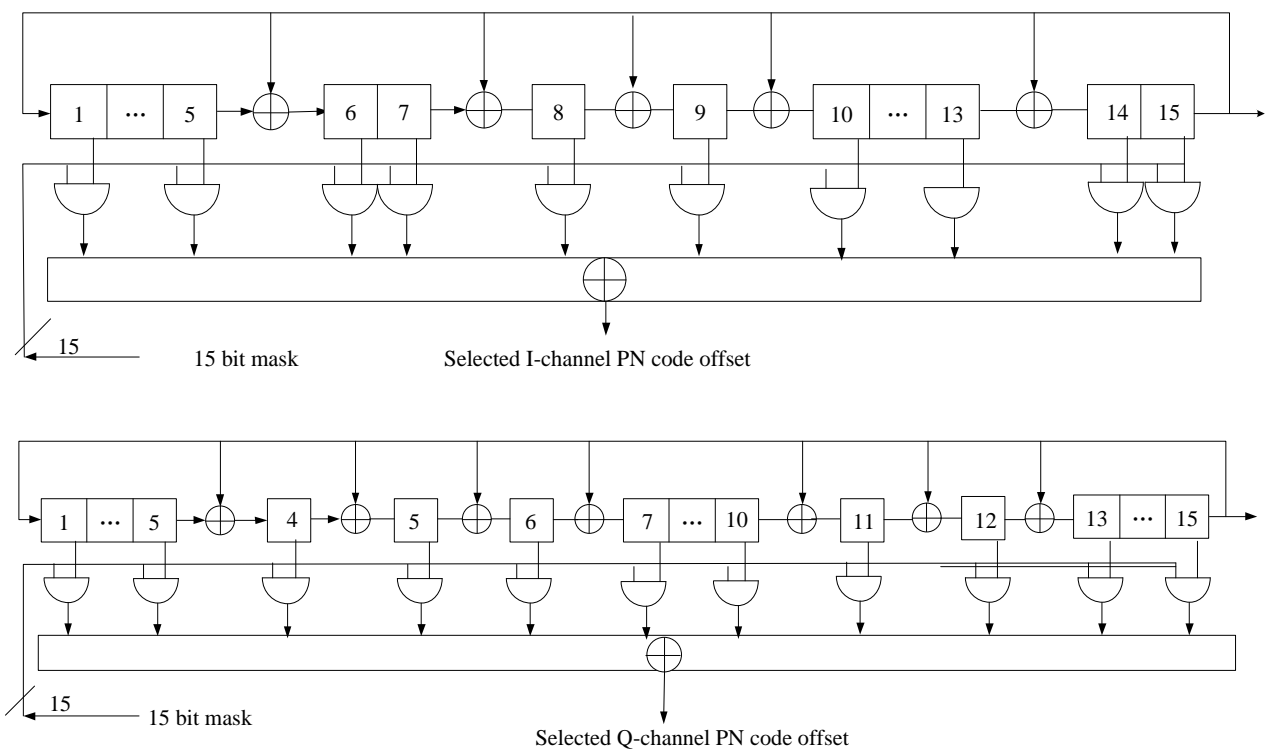

Figure 6 I, Q branch short code generator

\subsubsection{Baseband Filtering}

In this TT\&C system, we use the baseband shaping filter to meet the frequency response of to limit $\mathrm{S}(\mathrm{f})$, that is, passband $(0 \leq \mathrm{f} \leq \mathrm{fp}=590 \mathrm{kHz})$ ripple is not greater than $1.5 \mathrm{~dB}$,stopband $(\mathrm{f}>\mathrm{Fs}=$ $740 \mathrm{kHz}$ ) attenuation is not greater than $40 \mathrm{~dB}$.

\subsection{Channel design}

Compared with other communication channels, UAV high-speed mobile channel is the most complex one. Complex, poor propagation conditions are characteristic of a wireless channel, which is determined by the way in which wireless communication is performed in motion.

The simplest model for analysis in the channel is the additive white Gaussian noise channel (AWGN). In the model shown in Fig. 7, the transmitted signal $\mathrm{s}(\mathrm{t})$ is aggravated by the additive Gaussian white noise process $n(t)$ The received signal $r(t)$ is expressed as:

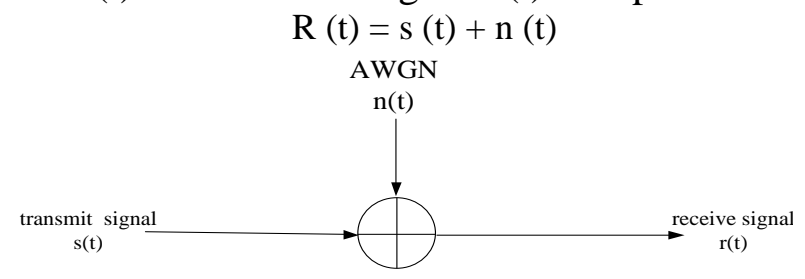

Figure 7 Additive white Gaussian noise channel

Multipath fading is due to the multipath transmission of mobile propagation environment caused by the decline, the amplitude generally obey the Rayleigh distribution or Les distribution, the rate of change is fast, is the fast fading loss. The multipath channel model, generally represented by the Rayleigh process. The Rayleigh process is obtained from the absolute value of the zero-mean complex Gaussian stochastic process. The first Rayleigh process is:

$$
\zeta_{l}(t)=\left|u_{l}(t)\right|=\left|u_{1, l}(t)+j u_{2, l}(t)\right|
$$

$\mathrm{u}_{1, l}(t), \quad \mathrm{u}_{2, l}(t)$ stand for two unrelated zero mean real Gaussian stochastic processes, the variance is expressed in the complex Gaussian process. According to Clarke's two-dimensional isotropic scattering model, the autocorrelation function is: 


$$
\mathrm{r}_{u_{i, l} u_{j, l}}(\tau)=\sigma_{l}^{2} J_{0}\left(2 \pi f_{\max } \tau\right)
$$

And where the $\mathrm{i}, \mathrm{j}=1,2, \mathrm{l}=1,2, \cdots \cdots, \mathrm{L}, \mathrm{f}_{\max }$ stands for Maximum Doppler frequency, $\mathrm{J}_{0}(\bullet$ ) stands for Zeroth order Bessel function of the first kind.

When the mobile channel is a generalized stationary non-correlated scattering (WSSUS) channel, the channel parameters on different delay paths are independent of each other, and the crosscorrelation function of any pair of complex Gaussian random processes is zero.

According to the central limit theorem, a large number of appropriately weighted sine wave superposition can approximate Gaussian stochastic process, so the real Gaussian process of Eq. (8) can be expressed as:

$$
\widetilde{\mathrm{u}}_{\mathrm{i}, \mathrm{l}}(t)=\sum_{n=1}^{N_{i, l}} c_{i, n, l} \cos \left(2 \pi f_{i, n, l} t+\theta_{i, n, l}\right)
$$

Where, $\mathrm{N}_{\mathrm{i}, 1}$ said the number of harmonics, $\mathrm{c}_{\mathrm{i}, \mathrm{n}, \mathrm{l}}$ said Doppler coefficient, $\mathrm{f}_{\mathrm{i}, \mathrm{n}, \mathrm{l}}$ said Doppler frequency, $\theta_{i, n, l}$ is the Doppler phase. These Doppler parameters are determined at initialization, so equation (10) gives a deterministic process. The autocorrelation function is:

$$
\widetilde{r}_{u_{i, l} u i, l}(\tau)=\sum_{n=1}^{N_{i, l}} \frac{c^{2}{ }_{i, n, l}}{2} \cos \left(2 \pi f_{i, n, l} t\right)
$$

The cross-correlation function of the two sine-wave signals of the same frequency is the same periodic signal with the same frequency as the original signal. If all the frequencies of any two random processes given by Eq. (10) are not equal, the cross-correlation function is:

$$
\tilde{\mathrm{r}}_{u_{i, l} u_{k, \lambda}}(\tau)=0, \mathrm{f}_{i, n, l} \neq \pm f_{k, m, \lambda}
$$

Where $\mathrm{i}, \mathrm{k}=1,2,1, \lambda=1,2, \ldots, \mathrm{L}$, and $\mathrm{i}=\mathrm{k}$ and $\mathrm{l}=\lambda$ are not simultaneously established. Thus, if different random processes are uncorrelated, their corresponding Doppler frequency sets must be disjoint [7].

\subsection{Receiver design}

The receiver section receives the signal from the channel, outputs the demodulated signal by baseband filtering, short code despreading, Walsh demodulation, descrambling, deinterleaving and convolution decoding. Due to the shadow effect and the influence of multipath fading, a large number of errors occur when the channel fading is large, resulting in a decrease in system performance. Diversity is an effective way to compensate for channel fading. By combining the independent fading channels with multiple copies of the same information, these signals are properly merged at the receiving end, which can be used at lower transmission power or bandwidth The cost of reducing the impact of fading improves communication quality. We use Rake to receive technology, which is an effective means of anti-multipath interference. Design the receiver in the multi-path number of 3 when the block diagram shown in Figure 8: 


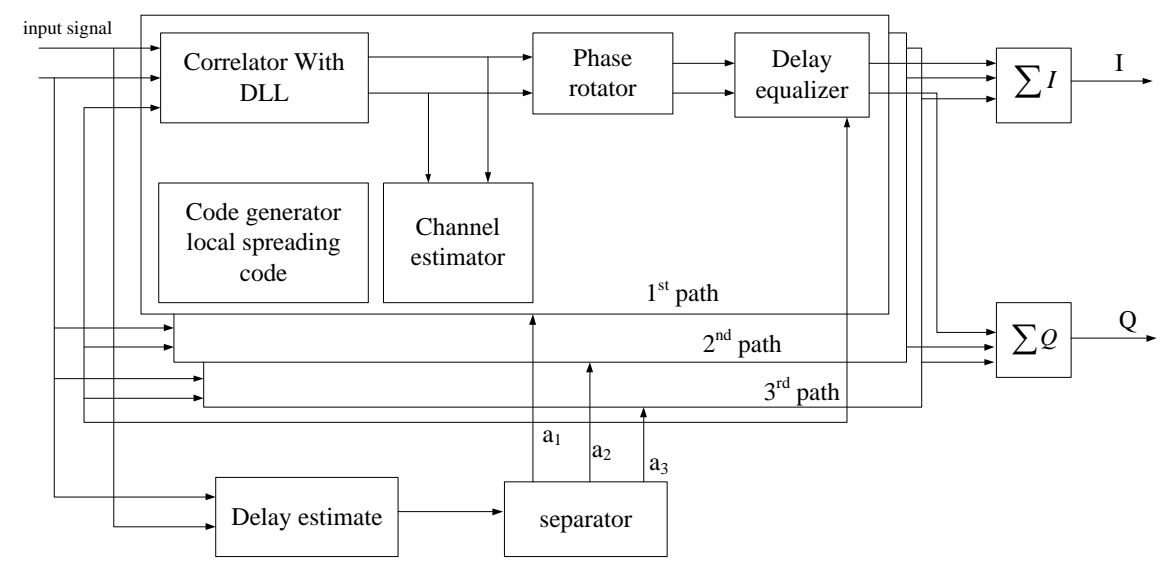

Figure 8 Rake receiver implementation block diagram

Each delay in the graph is done once and weighted, and then summed up to get an estimate of the information symbol and sent to the constellation demodulator. The operation performed by the correlator with the DLL is

$$
\sum_{n=1}^{Q} c_{n}^{(1)} y[n+D]
$$

Where $\mathrm{D}$ is the tap number corresponding to this delayed output, and $\mathrm{cn}$ is a constant. Set the number of multipaths in the scene to 3 , when the Rake receiver technology will multipath channel conditions, bandwidth is greater than the channel coherent bandwidth of the broadband signal to achieve the time to distinguish between different multipath components, the multi-path component separation, phase calibration After the synthesis of the final output signal.

Commonly used merging methods have maximum ratio combining (MRC) and equal gain combining (EGC), the maximum ratio of the merger is the need to change the weight coefficient of the value, for the actual system, this is very difficult to do. We use equal gain merger, equal gain is the weight coefficient is set to constant, that is to say that each branch component has the same weight coefficient, that is, the figure:

$$
\mathrm{a} 1=\mathrm{a} 2=\mathrm{a} 3=\sqrt{3}
$$

EGC merger does not need to estimate the signal-to-noise ratio of each branch, only need to a1 as the weight of the branch of the branch will merge.

\subsection{System performance simulation}

Based on the design of the monitoring and control link system described above, the BER performance of the QPSK system in the AWGN multipath channel with multipath effect is simulated and compared with the BER performance of the Rake receiver system. The performance of the system can be simulated as shown in Figure 9. 


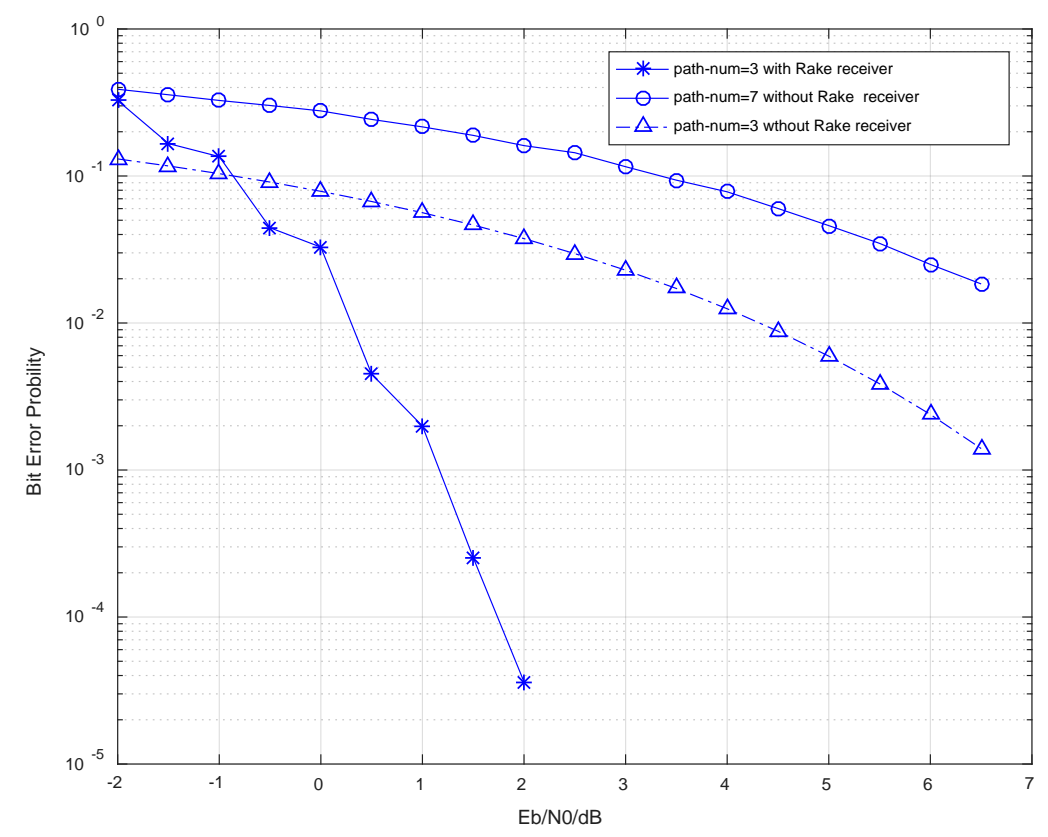

Figure 9 System performance simulation

The simulation parameters are set to the data rate of $9600 \mathrm{bps}$, the spreading chip rate is 1.2288 Mcps, the matched filter type adopts the cosine filter, the channel coding uses the convolutional code of $r=1 / 2, k=9$, using the 64th order Walsh matrix To distinguish between the various channels, QPSK two branch spread PN sequence length of $215=32768$. It can be seen that multipath interference has great influence on the system bit error probability and bit error rate. When the signal-to-noise ratio is $6 \mathrm{~dB}$, the performance of the system is $10 \mathrm{~dB}$ when the system error performance is compared with the multi-path number of 7 The However, when using Rake diversity reception, the system error rate improved significantly, in the signal to noise ratio of $2 \mathrm{~dB}$ when the bit error rate has reached $10^{-5}$.

\section{Conclusions}

In this paper, a kind of TT\&C link is designed for the TT\&C system of UAV-Swarm, and the characteristics of code division multiple access are used to realize the TT\&C of high-volume UAV. Due to the influence of the wireless channel in motion, with the influence of noise and multipath fading, multipath fading can enhance the received signal power by Rake receiver technology, thus improving the reliability of the system transmission. Finally, after the simulation test, you can achieve a relatively low bit error rate, that the data link on the UAV-Swarm of unmanned aerial vehicle system to achieve effective monitoring and control.

\section{References}

[1] A Burkle,F segor,M Kollmann(2011) Jurnal of intelligent \& Robotic System,61(1):339-353.

[2] JJ Corner,GB Lamont(2004) Parallel Simulation of UAV Swarm Scenarios, Simulation Conference, 1:355-363.

[3] Jout Sensalo J. Semi-blind(1998) CDMA code estimation in downlink[C]. 48th IEEE Vehicular Technology Conference, Ottawa,: 1356-1360.

[4] Sidi Ropoulos, Giannakis G B,and BRO R.(2000) Blind PARAFAC receivers for DS-CDMA systems[J]. IEEE Transactions on Signal Processing, 48(3): 810-823. 
[5]Ottossont,Svenssona(1995). IEEE VTC’95.Vehicular Technology ConferenceChicago, USA:IEEE Proceedings Multi-rate schemes in DS/CDMA systems.

[6] Song Yucui, Pan Ming, Wang Danhui(2013). Simulation of LANMAR Protocol in MANET Network [J]. Modern electronic technology. (1): 49-50.

[7]Jin Shi,Zhang Xiaolin,Zhou Qi.(2004) Journal of Aeronautical,Statistical Model of UAV Communication Channel.25(1):62-65 